\title{
ENVIRONMENTAL STRESS, HETEROZYGOTE ADVANTAGE AND GENOTYPE-ENVIRONMENT INTERACTION IN ARABIDOPSIS *
}

\author{
D. G. PEDERSON \\ Department of Genetics, N.C. State University, Raleigh, North Carolina, \\ 27607, U.S.A.
}

Received 9.v.67

A BASIC property of any set of plants is the type of response shown to environmental stress. Phenotypic stability has been measured both as the variability within single environments and as the constancy of means over a range of environments. Only the latter case will be considered here. Within both allogamous and autogamous species there may exist individual races highly stable to one or more classes of environmental stress. However, at least for outbreeding species, it is generally maintained that a high degree of stability is most notably a property of heterozygotes. The situation for self-fertilising species is less clear, although there are indications that stability is again a function of heterozygosity for most classes of environmental stress, in particular that imposed by high temperature (Lewis, 1955; Griffing and Langridge, 1963).

A set of genotypes may therefore be characterised by its mean response to environmental stress. A further property of any set of genotypes grown in a range of environments is the magnitude of the genotype $\times$ environment interaction. Parsons (1959) using Drosophila melanogaster and Hull et al. (1963) using poultry, have obtained evidence suggesting that the interaction effect is invariably of a low magnitude for a set of genotypes with uniformly high heterozygosity.

In the present study the obligately self-fertilising plant Arabidopsis thaliana has been used to investigate these two aspects of genotype-environment interaction. Three generations of plants are considered, each with a characteristic level of heterozygosity, and results are given for the growth of these plants at various levels of four factors of the environment. These are temperature, light intensity, amount of available water, and nutrient concentration.

\section{(a) Material}

\section{Material and methods}

From a collection of 45 races of Arabidopsis thaliana (L.) Heynh. (Cruciferae) available at the Division of Plant Industry, CSIRO, Canberra, ten were selected at random and used as parents. The geographic origins of these races ranged from Lund, Sweden, to Martuba, North Africa. Flowers of

* Published with the approval of the Director of Research as Paper No. 2341 of the Journal Series of the North Carolina Agricultural Experiment Station, Raleigh, North Carolina. This investigation was supported in part by Public Health Service Research Grant GM 11546 from the Division of General Medical Sciences. The experimental work was carried out at the Botany Department, Australian National University, Canberra, while the author was in receipt of a Wheat Industry Research Council Studentship. 
Arabidopsis are small (approximately $3 \mathrm{~mm}$. long) and are invariably selfpollinated even though protogyny is not uncommon. Individual races may therefore be regarded as homozygous, and a high degree of homogeneity is expected within the collection of plants making up a single race.

Fifteen $F_{1}$ hybrids were produced, each race being used three times as a gamete parent. $F_{1}$ plants were grown in a glasshouse, allowed to selfpollinate, and the $\mathrm{F}_{2}$ generation was used in subsequent experimental work. At the same time hybridisation was carried out with the $F_{1}$ plants, such that each $F_{1}$ genotype was used once as a female parent and once as a male parent. Fifteen double-cross hybrids therefore resulted.

In summary, the experimental material comprised:

(i) 10 parent races, considered to be homozygous;

(ii) $15 \mathrm{~F}_{2}$ hybrids, with an average heterozygosity of 0.5 ; and

(iii) 15 double-cross or $\left(\mathrm{F}_{1} \times \mathrm{F}_{1}\right)$ hybrids.

The theoretical study of Pederson (1966) may be applied to obtain an approximate estimate of 0.9 for the average degree of heterozygosity of the double-cross hybrids.

In the discussion of the results, the individual $\mathrm{F}_{2}-$ and $\left(\mathrm{F}_{1} \times \mathrm{F}_{1}\right)-$ crosses have often been referred to as " genotypes". Although this is strictly not the correct usage of the term, many difficulties were found to arise if alternative terms were introduced in the phrasing of conclusions.

\section{(b) Environment}

The aseptic culture method of Langridge (1957) was used throughout. The plants were grown singly on a nutrient-supplemented agar medium in 6 in. $\times \frac{5}{8}$ in. test-tubes, so that a high degree of repeatability was possible. Control of the external environment was obtained by growing the plants in an LB-type growth cabinet (Morse and Evans, 1962). The plant-growing space of 17.6 sq. $\mathrm{ft}$. allowed for a total capacity of 800 plants in tubes. To decrease the rate of desiccation of the growth-medium, particularly at high temperatures, the relative humidity within the cabinet was maintained at above 90 per cent. by introducing steam into the circulating air-stream.

Four major components of the natural environment were given primary consideration, namely temperature, light intensity, amount of available water, and nutrient concentration. For each of these factors three levels were chosen, namely the level resulting in optimal plant-growth and two levels reducing the growth-rate to approximately one-half and one-quarter of the optimum value respectively. The required levels were determined in a series of pilot experiments to give the complete set of nine test-environments (table 1).

A moisture-stress was simulated by the addition of mannitol to the growthmedium. This has the effect of increasing the osmotic pressure of the medium, thus inhibiting the uptake of water by plant tissues. The temperature was measured by means of a copper-constantan thermistor pushed into the agar of a single test-tube, and the light intensity within the cabinet was measured at the commencement of each trial at a level immediately above the test-tubes. The concentration of nutrients specified refers to both the macro- and micro-nutrients. The four types of stress produced quite distinct effects on plant morphology, and the existence of a common physiological basis for the stress-responses is therefore unlikely. 
Within each test-environment there were grown 20 plants of each parental race, 30 plants of each $F_{2}$ hybrid, and 10 plants of each double-cross hybrid. Although the plants were arranged in five randomised blocks, the betweenblock differences were found to be consistently non-significant. The data have therefore been analysed as though plants were distributed at random over the growing area, and the variation within genotypes has been used as an estimate of error. For the two segregating generations this estimate is inflated to some extent by a genetic component, and further consideration will be given to this point in the section which follows.

TABLE 1

$A$ list of the nine test-environments which were employed for growth of the parental, $F_{2}$, and double-cross material. (The symbols " $T$ ", " $L$ ", " $M$ " and " $\mathcal{N}$ " refer to temperature, light intensity, mannitol concentration, and nutrient concentration respectively, and "medium" and "high" indicate the level of stress)

\begin{tabular}{|c|c|c|c|c|}
\hline \multirow[b]{2}{*}{$\begin{array}{c}\text { Type of } \\
\text { environment }\end{array}$} & \multicolumn{4}{|c|}{ Environmental component } \\
\hline & $\begin{array}{c}\text { Temperature } \\
\left({ }^{\circ} \mathrm{C} .\right)\end{array}$ & $\begin{array}{l}\text { Light intensity } \\
\text { (f.c.) }\end{array}$ & $\begin{array}{c}\text { Mannitol } \\
\text { (gm./gm. medium) }\end{array}$ & $\begin{array}{c}\text { Nutrient } \\
(\text { standard }=1)\end{array}$ \\
\hline Optimum & 25 & 2000 & 0 & 1 \\
\hline Medium T & 29 & 2000 & 0 & 1 \\
\hline High $\mathrm{T}$ & 31 & 2000 & 0 & 1 \\
\hline Medium L & 25 & 900 & 0 & 1 \\
\hline High L & 25 & 400 & 0 & 1 \\
\hline Medium M & 25 & 2000 & $0 \cdot 0125$ & 1 \\
\hline High M & 25 & 2000 & $0 \cdot 02$ & 1 \\
\hline Medium N & 25 & 2000 & 0 & $\frac{1}{12}$ \\
\hline High N & 25 & 2000 & 0 & $\frac{1}{36}$ \\
\hline
\end{tabular}

Growth was allowed to proceed for 14 days from the time of germination, after which the plants were individually pulled from the agar medium, their roots blotted, and the fresh weights measured to the nearest tenth of a milligram. This was the only plant character recorded. Prior to analysis, all fresh weights were logarithmically transformed since they had been derived from plants in the exponential phase of growth.

\section{RESUlts}

For each trial the set of fresh-weight data was incomplete as a result of poor seed germination or the loss of plants through failure of the cotyledons to emerge from the agar medium. Analyses have therefore been based on the genotype means, and pooled estimates of error have been derived for analyses of variance.

\section{(a) Generation $\times$ environment interaction}

The set of means for each of the nine trials, together with their standard errors and the number of observations involved, are given in table 2. There is evidence of positive heterosis in that the mean growth rate within a trial was always greatest for the most heterozygous generation. Although each of the generations showed a decrease in mean growth-rate with increasing stress, there is some indication that the effects were not equal for the three generations. A two-factor analysis of variance has therefore been carried out on the complete set of data for each class of stress to test for the significance of generation $\times$ environment interactions. The main effects are those due 
to generations and environments, and genotypes are nested within generations. The mean squares from these analyses will not be presented, since with one exception the between-generation differences and the generation $\times$ environment interactions are significantly different from zero at the $0 \cdot 1$ per cent. level. The one exception is for the case of interaction involving a

TABLE 2

The generation means ( $\log _{10} \mathrm{mg}$.) and their standard errors for the set of nine trials; numbers in brackets indicate the number of observations involved in each mean

Environmental factor

Temperature

Light intensity

Mannitol concentration

$\mathrm{F}_{2}$ $\mathrm{F}_{1} \times \mathrm{F}_{1}$

Nutrient concentration

Environmental level

$\begin{array}{lccc}\text { Generation } & \text { Optimum } & \text { Medium stress } & \text { High stress } \\ \text { Parent } & 1 \cdot 533 \pm 0.006(173) & 1 \cdot 214 \pm 0 \cdot 016(188) & 0.519 \pm 0.066(117) \\ \mathrm{F}_{2} & 1 \cdot 600 \pm 0 \cdot 005(441) & 1 \cdot 390 \pm 0 \cdot 007(445) & 0 \cdot 765 \pm 0.016(427) \\ \mathrm{F}_{1} \times \mathrm{F}_{1} & 1 \cdot 627 \pm 0.008(142) & 1 \cdot 499 \pm 0.012(147) & 1 \cdot 151 \pm 0.030(144)\end{array}$

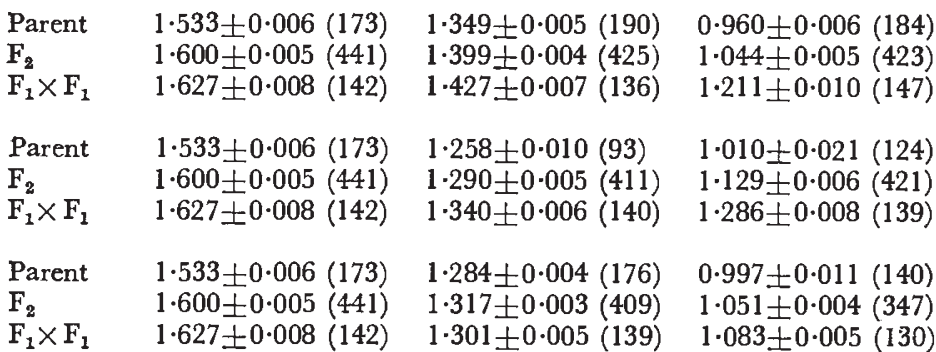

TABLE 3

Estimated components of variance obtained from two-way analyses of variance; components are given as both absolute values and percentages of the total variance

\begin{tabular}{lcccc}
\multicolumn{4}{c}{ Environmental factor } \\
Variance component & Temperature & Light intensity & $\begin{array}{c}\text { Mannitol } \\
\text { concentration }\end{array}$ & $\begin{array}{c}\text { Nutrient } \\
\text { concentration }\end{array}$ \\
Generations & $\begin{array}{c}0.0255 \\
(28.0)\end{array}$ & 0.0044 & 0.0051 & 0.0010 \\
& $(28.6)$ & $(26.4)$ & $(12.6)$ \\
Generation $\times$ environment & 0.0186 & 0.0025 & 0.0028 & 0.0004 \\
& $(20.5)$ & $(16.3)$ & $(14.3)$ & $(4.7)$ \\
Error & 0.0468 & 0.0085 & 0.0114 & 0.0062 \\
& $(51.5)$ & $(55.1)$ & $(59.3)$ & $(82.7)$ \\
Sum & 0.0909 & 0.0154 & 0.0193 & 0.0076
\end{tabular}

nutrient stress, which is significant only at the 1 per cent. level. But expressions for the expectations of mean squares assuming all effects to be fixed have been used to estimate components of variance, and the values for components due to between-generation differences, generation $\times$ environment interaction, and error are given in table 3. There is an apparent grouping into two classes, with the results for the temperature, light intensity, and mannitol concentration trials forming a relatively homogeneous set and 
the nutrient trial differing from these in that a major part of the total variance is attributable to error.

The effects of the stresses may be further investigated by considering the differences in mean between the $F_{2}$ and parent generations, and between the double-cross and $F_{2}$ generations. The values for these differences are set out in table 4 , and the variance ratio accompanying each value indicates

TABLE 4

The effects of environmental stress on the two measures of heterozygote advantage, viz. the $\left(F_{2}-\right.$ midparent $)$ differential and the (double-cross $\left.-F_{2}\right)$ differential $\left(\log _{10} \mathrm{mg}\right.$.)

\begin{tabular}{|c|c|c|c|c|c|}
\hline \multirow[b]{2}{*}{ Environmental factor } & \multirow{2}{*}{$\begin{array}{l}\text { Level of } \\
\text { stress }\end{array}$} & \multicolumn{2}{|c|}{$\mathrm{F}_{2}-\mathrm{MP}$} & \multicolumn{2}{|c|}{$\left(F_{1} \times F_{1}\right)-F_{2}$} \\
\hline & & value & $F(1,23)$ & value & $F(1,23)$ \\
\hline \multirow[t]{3}{*}{ Temperature } & 一 & $0 \cdot 067$ & $5 \cdot 1 *$ & 0.028 & $1 \cdot 3 \mathrm{NS}$ \\
\hline & medium & $0 \cdot 176$ & $9 \cdot 6 * *$ & $0 \cdot 109$ & 4.7* \\
\hline & high & $0 \cdot 246$ & $37 \cdot 5 * * * *$ & $0 \cdot 386$ & $69 \cdot 5 * * *$ \\
\hline \multirow[t]{3}{*}{ Light intensity } & - & $0 \cdot 067$ & $5 \cdot 1 *$ & $0 \cdot 028$ & $1 \cdot 3 \mathrm{NS}$ \\
\hline & medium & 0.050 & $5 \cdot 0 *$ & $0 \cdot 028$ & $3 \cdot 6 \mathrm{NS}$ \\
\hline & high & $0 \cdot 084$ & $5 \cdot 8 *$ & $0 \cdot 167$ & $40 \cdot 7 * * * *$ \\
\hline \multirow[t]{3}{*}{ Mannitol concentration } & - & 0.067 & $5 \cdot 1 *$ & $0 \cdot 028$ & $1 \cdot 3 \mathrm{NS}$ \\
\hline & medium & $0 \cdot 033$ & $0.4 \mathrm{NS}$ & $0 \cdot 050$ & $7 \cdot 7 * *$ \\
\hline & high & $0 \cdot 120$ & $9 \cdot 7 * *$ & $0 \cdot 157$ & $51 \cdot 1 * * *$ \\
\hline \multirow[t]{3}{*}{ Nutrient concentration } & - & 0.067 & $5 \cdot 1^{*}$ & $0 \cdot 028$ & $1 \cdot 3 \mathrm{NS}$ \\
\hline & medium & $0 \cdot 033$ & $2 \cdot 9 \mathrm{NS}$ & $-0 \cdot 016$ & $1 \cdot 8 \mathrm{NS}$ \\
\hline & high & 0.054 & $6 \cdot 5 *$ & $0 \cdot 032$ & 7.3* \\
\hline
\end{tabular}

NS $=$ not significant.

*,** and *** indicate significance at the 5,1 and $0 \cdot 1$ per cent. level respectively.

its significance. The between-generation differences increased most significantly for stress imposed by high temperature, while similar, though less significant, increases were produced by the light intensity- and mannitolstresses. Finally, there was only a slight tendency for the between-generation differences to increase with increasing nutrient stress.

\section{(b) Genotype $\times$ environment interaction}

In the previous section interactions with environment were considered at the generation level, and the average phenotypic stability of a generation was found to be directly related to its mean level of heterozygosity. Consideration will now be given to gene-environment interaction at the genotype level.

Consider first the results for growth at three temperatures. A one-way analysis of variance has been carried out on the data for each genotype, and the between-temperatures component estimated from expected mean squares. These estimates of between-environment variation will be used as measures of phenotypic stability. The overall mean for each genotype at the three temperatures has also been calculated, and the joint distribution of stabilities and overall means is plotted in fig. 1, a. The data for growth at three light intensities, three mannitol concentrations, and three nutrient concentrations have been similarly analysed, and the joint distributions for 
these remaining environmental factors are given in fig. 1, b-d. In interpreting these figures, it is important to note that phenotypic stability was measured as the macro-environmental component of variance, so that the genotypes with the lowest values are the most stable.
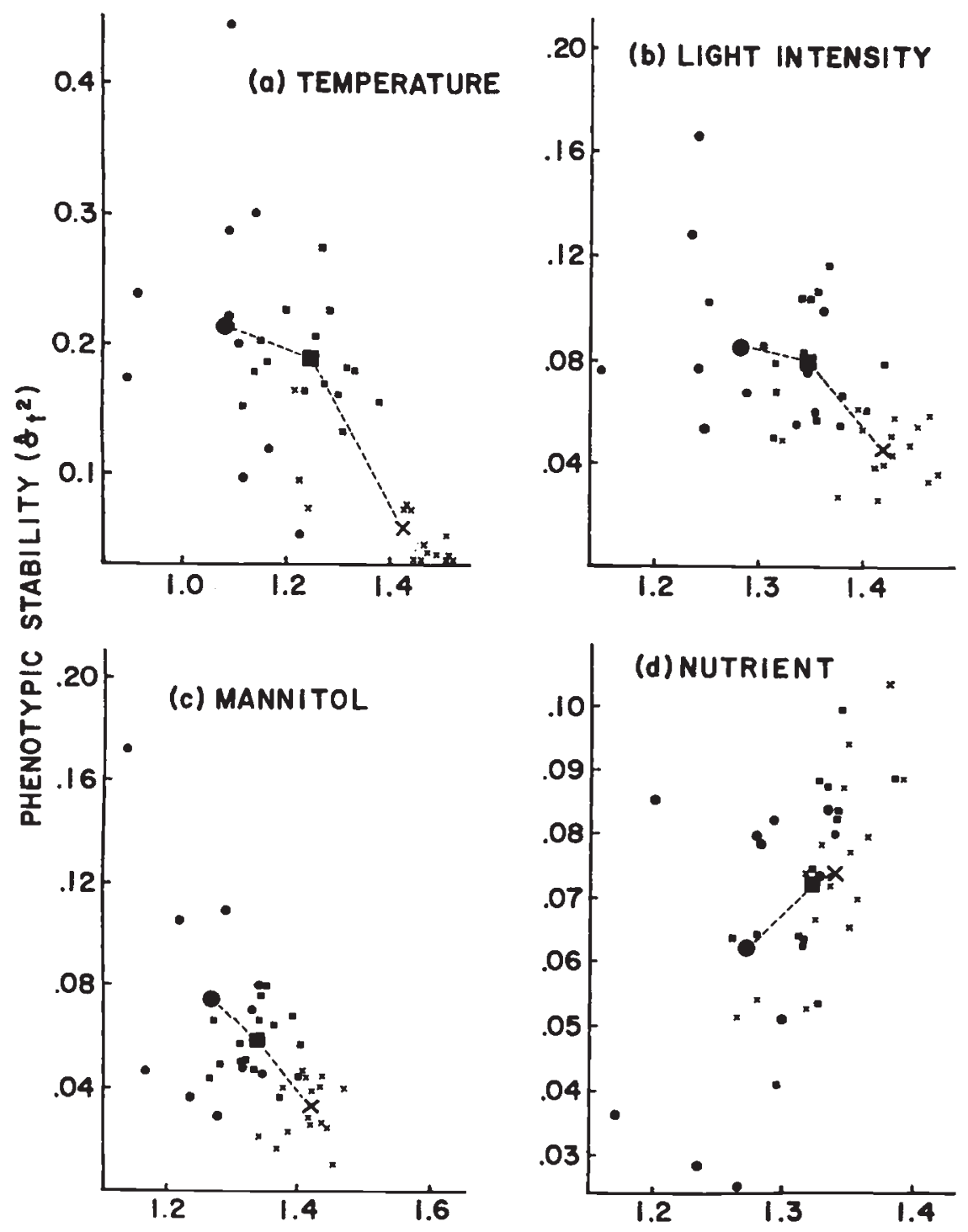

\section{MEAN OVER THREE LEVELS OF STRESS ( $\left.\log _{10} \mathrm{mgms}\right)$}

FIG. 1.-Joint distributions of phenotypic stability $\left(\hat{\sigma}_{t}^{2}\right)$ and overall mean $[\log$ (fresh weight in $\mathrm{mg}$.)] for the parent races $(\bullet), \mathrm{F}_{2}$ 's $(\boldsymbol{\square})$ and double-crosses $(\mathrm{X})$. The mean value for each generation is represented as a larger symbol.

The larger symbols in fig. 1, a-d are plots for the grand means and mean stabilities of the respective generations. The fact noted in the previous section is clearly illustrated by the positions of these symbols, namely that 
both the generation mean and the degree of stability decrease in proportion to the mean level of heterozygosity for stress imposed by high temperature, low light intensity, and high mannitol concentration. For the nutrient stress there is an apparent opposite tendency, in that the mean stability increases with a decrease in heterozygosity.

However, there is a more significant observation to be made from fig. 1, a-d. For each class of stress the double-crosses consistently form a homogeneous group with respect to both overall mean and phenotypic stability, while at the other extreme the parental values are scattered over a wide range. This result suggests that the double-crosses are more uniform as a group than the other generations of plants. A two-factor analysis of

TABLE 5

Mean squares for genotype $\times$ environment interaction and error, together with the variance ratios testing for significance of the interaction effects

Environmental factor

Temperature

Light intensity

Mannitol concentration

parent

$\mathrm{F}_{2}$

$\mathrm{F}_{1} \times \mathrm{F}_{1}$

Nutrient concentration
Mean square

$\overbrace{\begin{array}{c}\text { Genotype } \times \\ \text { environment }\end{array} \text { Error }}^{\text {Mronmen }}$

$\begin{array}{ll}0 \cdot 0075 & 0 \cdot 0047 \\ 0 \cdot 0032 & 0 \cdot 0015 \\ 0 \cdot 0050 & 0 \cdot 0056\end{array}$

$0 \cdot 0004$

$0 \cdot 0003$

$0 \cdot 0011$

$0 \cdot 0017$

$0 \cdot 0011$

$0 \cdot 0019$

0.0004

$0 \cdot 0010$

$0 \cdot 0012$

$0 \cdot 0008$

$0 \cdot 0028$

0.0012

$0 \cdot 0007$
$0 \cdot 0007$

0.0002

$0 \cdot 0006$
Variance ratio

$\mathrm{F}(18,448)=1 \cdot 6 *$

$\mathrm{F}(28,1268)=2 \cdot 1 * * *$

$\mathrm{F}(28,388)=0.9 \mathrm{NS}$

$\mathrm{F}(18,517)=9 \cdot 8 * * *$

$\mathrm{F}(28,1243)=5 \cdot 5 * * *$

$\mathrm{F}(28,380)=1 \cdot 0 \mathrm{NS}$

$\mathrm{F}(18,360)=2 \cdot 5 * * *$

$\mathrm{F}(28,1228)=2 \cdot 6 * * *$

$\mathrm{F}(28,376)=1.5 *$

$F(18,459)=4 \cdot 3 * * *$

$\mathrm{F}(28,1152)=5 \cdot 2 * * *$

$\mathrm{F}(28,363)=1 \cdot 1 \mathrm{NS}$

NS = not significant.

*, ** and *** indicate significance at the 5,1 and $0 \cdot 1$ per cent. level respectively.

variance, with genotypes and environmental levels as the main factors, has therefore been carried out on each of the 12 combinations involving one generation of plants and a particular environmental factor. For each analysis the main effects are significantly different from zero at the 1 per cent. level. The mean squares for these factors will therefore not be presented, but in table 5 there are set out the mean squares for genotype $\times$ environment interaction and error, together with the appropriate degrees of freedom for the variance ratio $(F)$. It was noted previously that for each of the segregating generations the estimate of error includes a genetic component. But the F-test is still a valid test of significance since the component of variance due to genetic segregation appears in the expectations of both the interaction and error mean squares.

For the set of double-cross hybrids the interaction mean square is not significantly different from zero for variation in temperature, light intensity, or nutrient concentration, and is significant only at the 5 per cent. level for the mannitol treatment. It is therefore apparent that the double-crosses 
generally form a homogeneous set with respect to phenotypic stability. By way of contrast, the interaction for the set of parent genotypes is significant at the $0 \cdot 1$ per cent. level in three of the cases, and significant at the 5 per cent. level for the remaining case of a temperature stress.

It would be concluded from the F-tests that a high interaction effect is common to the $F_{2}$ and parental generations. However, components of variance have been estimated on the basis of random genotypic and fixed environmental effects, and the values for these (table 6) show that the interaction effect is always greater for the parent generation. In general, it can

TABle 6

Components of variance attributable to genotypic differences $\left(\hat{\sigma}_{o}^{2}\right)$, genotype $\times$ environment interaction $\left(\hat{\sigma}_{g t}^{2}\right)$, and error $\left(\hat{\sigma}_{e}^{2}\right)$

Environmental factor

Generation

Component of variance

Temperature

Light intensity

Parent

$\mathrm{F}_{2}$

$\mathrm{F}_{1} \times \mathrm{F}_{1}$

0.00894

0.00543

0.00897

\begin{tabular}{|c|c|}
\hline$\hat{\sigma}_{g}$ & $\hat{\sigma}_{a t}^{2}$ \\
\hline
\end{tabular}

Parent
$\mathrm{F}_{2}$
$\mathrm{~F}_{1} \times \mathrm{F}_{1}$

$0 \cdot 00468$

0.00170

0.00281

$0 \cdot 04326$

0.05501

0.00176

$0 \cdot 00000$

0.04586
0.05395

0.05305

0.06292

Mannitol concentration

Parent

0.00380

0.00140

0.00627

0.01475

0.00117

0.00000

0.01023

(2012

$F_{2}$
$F_{1} \times F_{1}$

$0 \cdot 00479$

0.00298

$0 \cdot 01551$

0.01140

0.00183
0.00100

0.00063

0.01138

0.02328

0.00041

0.00752

0.01384

Parent

0.00254

$0 \cdot 00217$

0.00778

0.00893

Nutrient concentration

$\mathrm{F}_{2} \times \mathrm{F}_{1}$

$0 \cdot 00078$

0.00094

0.00580

$0 \cdot 01249$

0.00089

$0 \cdot 00006$

$0 \cdot 00552$

0.00752

0.00647

be said that the magnitude of the genotype $\times$ environment interaction decreases from the parent generation to the double-cross generation, with the $\mathrm{F}_{2}$ generation occupying an intermediate position. Again it may be noted that the magnitude of the interaction component is independent of the effects of segregation in the $\mathrm{F}_{2}$ and double-cross generations. Furthermore, in two instances the error component is greater for the homozygous parent generation than for either of the segregating generations, and it would therefore appear that no reasonable allowance may be made for the effects of genetic segregation.

The component of variance attributable to genotypes is a pooled measure of the between-genotype variation within single environments. It does not have a constant value for the three generations, but is invariably greatest for the most homozygous material (table 6).

\section{Discussion}

The heterozygote superiority observed to exist under all environmental regimes was not unexpected, as a similar phenomenon has been observed for a great number of plant and animal characters. A sufficient explanation may be given in terms of the dominance effects of single loci (e.g. Falconer, 1960, p. 257). 
With regard to the effect of environment on heterozygote superiority, Jinks and Mather (1955) analysed data obtained in three successive years for a diallel set involving eight Nicotiana rustica varieties. Plot means were measured for the characters plant height, flowering time, and leaf length, but only for the first character were the parental and $F_{1}$ generations observed to differ in average stability over the three years. The hybrid material was the less stable. In the study reported by Bucio Alanis (1966), plant heights were measured over a period of 16 years for two varieties of $\mathcal{N}$. rustica and their hybrid. The variation from year to year was partitioned into its component parts, and the magnitude of genotype $\times$ environment interaction was found to be directly proportional to the environmental effect. The degree of heterosis, when measured as the excess of the hybrid over the superior parent, was observed to increase as the environment became less favourable, but at the same time there was a decrease in the superiority of the hybrid over the mid-parent value.

A result more like that of the present study was obtained by Lewis (1955), who constructed the hybrid between Lycopersicum esculentum and $L$. pimpinellifolium and then measured the parental and hybrid growth rates at various levels of light intensity and temperature. Both parents were affected by variation in the environmental conditions, but the hybrid growth rate was found to be relatively invariant. Griffing and Langridge (1963) have previously demonstrated the existence of temperature-dependent heterosis in Arabidopsis, and in this regard the present study does little but corroborate their findings.

Finlay (1963) carried out a field trial in which ten varieties of barley and their $45 \mathrm{~F}_{2}$ hybrids were grown at a single site in three successive years. In each year the mean grain yield was higher for the hybrid material, and the degree of heterozygote advantage was greatest for the year least favourable to plant growth.

In each of the studies cited, a predominantly self-fertilising species was used to examine the relationship between heterozygote advantage and environment, but there is a lack of any real uniformity in the results. A significant factor may be the range of environments considered in each case. The characters measured by Jinks and Mather showed little deviation over the three years, and although a somewhat greater range of environments was considered by Bucio Alanis it should be noted that the range was extended at the "favourable" end of the scale by a change in the culture conditions for the final years of the experiment. By way of contrast, in the present study and that of Finlay the most severe environments reduced the average fresh weight and yield respectively to about one-quarter of their maximum test values. It is therefore possible that only the most severe of stresses will induce a marked increase in heterozygote advantage for an autogamous species. But the results for various nutrient concentrations in the present study indicate that even for such conditions of severe stress no general statement can be made.

In a discussion of temperature-dependent heterosis as observed in both Arabidopsis and Drosophila, Langridge (1962) suggested that alleles sensitive to high temperature are responsible for the production of unstable enzymes, with mutant expression occurring only at elevated temperatures due to their high thermal coefficient for inactivation. The assumption was made that, under stress conditions, a non-sensitive allele can produce sufficient normal 
enzyme in the heterozygous condition to compensate for the presence of the temperature-sensitive allele. A hybrid would therefore exhibit superior stability if its parents were homozygous for sensitive alleles at different loci. An explanation based on the existence of alleles specifying stress-sensitive products would also be suitable for the effects of a low light intensity and a high mannitol concentration as obtained in the present study.

On the basis of this explanation, it is clear that a unique response of heterozygote advantage to environment would be expected for each set of parents and their hybrids. In particular, few stress-dependent alleles would be expected in a set of inbred genotypes chosen for their high level of stability, and correspondingly no significant relationship would be expected between heterosis and environment. It may be noted that the parents used in the present study were chosen at random from a world collection of Arabidopsis races, and great variation was observed in their stabilities to various environmental stresses. In a similar fashion the barley varieties used by Finlay exhibited a wide range of adaptation to the test conditions, thus indicating the presence of alleles with various degrees of sensitivity to unfavourable environments.

In the second section of the experimental study estimates were made of the genotype $\times$ environment interaction within groups differing in average heterozygosity. Hull and Gowe (1962) have proposed that significant genotype $\times$ environment interaction effects are commonly associated with large between-genotype differences. In their study, half-sib groups of poultry were compared in two separate sets of environments, and interactions with environment were found to be greatest for traits with a large between-group genetic variance. A further study was made, comparing the interactions of pure strains and strain crosses of poultry with two types of environment (Hull et al., 1963). Several traits were tested in four successive years, but only two were affected to any extent by environment, namely body-weight at housing and age at sexual maturity. Significant interaction effects were observed for these traits alone, and in each case the between-group variance and the strain $\times$ environment interaction effects were greater for the pure strains than for the cross strains. Temperature studies with both Arabidopsis (Griffing and Langridge, 1963) and Drosophila (Parsons, 1959) have shown that homozygous lines exhibit a greater genotype $\times$ environment interaction than crosses between lines.

The effect observed in the present study has therefore been previously obtained in experiments using both plant and animal material. Accordingly, consider the general situation in which a number of genotypes are grown under a range of environments. The available evidence indicates that between-genotype effects and genotype $\times$ environment interaction effects are not independent, and that, in particular, hybrid genotypes resulting from inter-crossing within an originally inbred population are likely to exhibit lower between-genotype variation and a less significant interaction effect than the parents. This phenomenon is independent of the nature of the single-locus gene action, whether predominantly additive or non-additive, and therefore is unrelated to stress-dependent heterosis.

In recent years there has been a great deal of speculation on the commercial use of hybrids in autogamous plant species, and the results of the present study are of interest since they indicate a general superiority of hybrid material, particularly in stress environments. However, the general 
conclusion from this and other studies is that the stability shown by a hybrid, relative to that of its parents, depends less upon its level of heterozygosity than upon the nature of the parental genotypes and the response characteristics of their constituent alleles. The models which are sufficient to explain the phenomenon of stress-dependent heterosis imply that homozygous genotypes may be found which are equally as stable as any hybrid, and it is true that in the present study there did exist homozygous races of comparable stability to the majority of the hybrid genotypes. But there is overall support for the suggestion of Allard and Bradshaw (1964), namely that until suitably stable homozygous varieties are produced then the use of hybrids should definitely be considered for commercial practice.

With the aseptic culture method plants were grown singly in test-tubes, and the character measured as "phenotypic stability" was therefore the capacity for "individual buffering" (Allard and Bradshaw, 1964). However, a double-cross is a mixture of genotypes and is therefore capable of exhibiting a high level of "populational buffering" when yrown in bulk. In addition, the relative genetic potentials of a number of double-cross hybrids may be accurately determined from relatively few trials due to their low interaction with environment. These factors suggest that if hybrid plants of an autogamous species were desired for commercial use, the most suitable material would be double-cross hybrids. The chief difficulty would appear to be the production of sufficient hybrid seed.

\section{Summary}

1. Ten races of the self-fertilising plant Arabidopsis thaliana were used in a hybridisation programme to produce $15 \mathrm{~F}_{2}$ hybrids and 15 double-cross hybrids.

2. The three generations were grown under a range of stress and nonstress environments involving variation in temperature, light intensity, amount of available water, and nutrient concentration. An aseptic culture method was used and all growth was carried out within a controlledenvironment growth cabinet, the character measured being fresh weight after 14 days' growth.

3. The degree of heterozygote advantage, as measured by the $\left(\left(F_{1} \times F_{1}\right)-F_{2}\right)$ and $\left(F_{2}-\right.$ parent $)$ differentials, increased with an increase in temperature-, light intensity-, and moisture-stress, but was relatively invariant under a range of nutrient concentrations.

4. Estimates were obtained for the interaction of genotypes with the three levels of each of the environmental factors.

5. The magnitude of the interaction effect was inversely related to the average heterozygosity of a generation, being always greatest for the parent races and least for the double-cross hybrids.

\section{REFERENGES}

AllARD, R. W., AND BRADSHAW, A. D. 1964. Implications of genotype-environment interaction in applied plant-breeding. Crop Sci., 4, 503-508.

BUCIO ALANIS, L. 1966. Environmental and genotype-environmental components of variability. 1. Inbred lines. Heredity, 21, 387-397. 2. Heterozygotes. Heredity, 21, 399-405.

FAlCONER, D. s. 1960. Introduction to Quantitative Genetics. Oliver and Boyd, Edinburgh and London. 
FINLAY, K. W. 1963. Adaptation-its measurement and significance in barley breeding. 1st Int. Barley Genetics Symp., Proc. Wageningen, pp. 351-359.

GRIFFING, B., AND LANGRIDGE, J. 1963. Phenotypic stability of growth in the self-fertilised species Arabidopsis thaliana. In Statistical Genetics and Plant Breeding: NAS-NRC, 982, 368-394.

HULL, P., AND GOWE, R. s. 1962. The importance of interactions detected between genotype and environmental factors for characters of economic significance in poultry. Genetics, 47, 143-159.

HULl, P., GOWE, R. S., SLEN, S. B., AND CRAWFORD, R. D. 1963. A comparison of the interaction, with types of environment, of pure strains or strain crosses of poultry. Genet. Res., 4, 370-381.

JINKs, J. L., AND MATHER, x. 1955. Stability in development of heterozygotes and homozygotes. Roy. Soc. (London), Proc., B, 143, 561-578.

LANGRIDGe, J. 1957. The aseptic culture of Arabidopsis thaliana (L.). Heynh. Aust. F. Biol. Sci., 10, 243-252.

LANGRIDGE, J. 1962. A genetic and molecular basis for heterosis in Arabidopsis and Drosophila. Amer. Nat., 96, 5-28.

LEWIS, D. 1955. Gene interaction, environment and hybrid vigour. Roy. Soc. (London), Proc., $B, 144,178-185$.

MORSE, R. N., AND EVANS, L. T. 1962. Design and development of GERES-an Australian phytotron. 7. Agric. Eng. Res., 7, 128-140.

PARSONS, P. A. 1959. Genotypic-environmental interactions for various temperatures in Drosophila melanogaster. Genetics, 44, 1325-1333.

PEDERSON, D. G. 1966. The expected degree of heterozygosity in a double-cross hybrid population. Genetics, 53, 669-674. 